\title{
Seroprevalence of Bordetella pertussis in healthcare workers in a Portuguese tertiary referral hospital
}

Braz M. C.', Menezes M. F. ", Lopes A. C.", Sousa M. J.", Neto A. S.'

I Pediatric Department, CUF Descobertas Hospital

" Germano de Sousa Group, Laboratory Medicine Centre

\section{Background:}

Methods:

Bordetella pertussis causes an acute infectious disease that, in newborns and little infants, can present with severe clinical manifestations or even death. The world prevalence is around 16 million cases per year, with 195.000 estimated deaths.

In Portugal, whole cell pertussis vaccine was introduced in 1965 followed, in 2006, by the acellular form included in a triple vaccine (diphteria, tetanus and pertussis). The national vaccine coverage is about $95 \%$ and the program includes 5 doses (2, 4, 6, 18 months, 5 years).

Yet a raising number of cases have been observed, with an incidence of 2.14 per 100.000 cases in 2012. The emergence of this disease in Portugal and in other countries can be related with multiple factors: better diagnostic tools (molecular techniques), genetic evolution of Bordetella pertussis and the switch from whole cell vaccine to the acellular form, that seems to give less protection. Duration of protection against Pertussis infection is unknown (after last immunization or disease).

Healthcare professionals, working with children, can be the source of infection of hospital outbreaks. Knowing the seroprevalence of pertussis antibodies among them is important to reinforce the need for vaccination in susceptible professionals.
Prospective study including healthcare workers from neonatology and pediatric departments and clinical pathology laboratory of a tertiary referral hospital (physicians, nurses, medical auxiliars, laboratory technicians, administrative assistants).

Demographic data (gender, age, professional category, department) and last date of Bordetella pertussis immunization (if possible) were collected. People with acute respiratory symptoms, pregnants or vaccinated with pertussis during the year of 2016 were excluded.

Seroprevalence for pertussis was tested through IgG for pertussis toxin by ELISA (Euroimmun ${ }^{\circledR}$ ) and it was considered positive $(>100 \mathrm{UI} / \mathrm{mL})$, negative $(<40 \mathrm{UI} / \mathrm{mL})$ or equivocal (40-100 UI/mL). The equivocal results were retested. Sensibility - 100\%; specificity $-95.5 \%$. This study was approved by the ethical committee of the hospital.

Table 1- Demographic Data by age

\begin{tabular}{|c|c|}
\hline Age groups (years) & \\
\hline $18-24$ & 4 \\
\hline $25-34$ & 27 \\
\hline $35-44$ & 34 \\
\hline$\geq 45$ & 25 \\
\hline Total & $\mathbf{9 0}$ \\
\hline
\end{tabular}
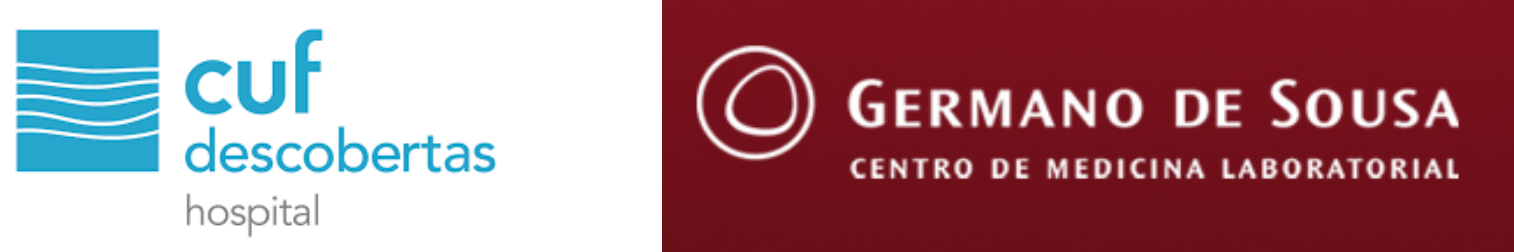

Table 2 - Demographic Data by professional category and department

\begin{tabular}{|c|c|c|c|c|c|c|}
\hline Prof. category & ER & Neo. Dep. & Cl. Practice & Ped . Dep. & Laboratory & Total \\
\hline Nurses & 12 & 9 & 3 & 3 & 0 & $\mathbf{2 7}$ \\
\hline Physicians & 9 & 5 & 3 & 7 & 2 & $\mathbf{2 6}$ \\
\hline Medical auxiliars & 9 & 6 & 4 & 1 & 1 & $\mathbf{2 1}$ \\
\hline Lab. technicians & 0 & 0 & 0 & 0 & 8 & $\mathbf{8}$ \\
\hline Administratives & 1 & 2 & 4 & 1 & 0 & $\mathbf{8}$ \\
\hline Total & $\mathbf{3 1}$ & $\mathbf{2 2}$ & $\mathbf{1 4}$ & $\mathbf{1 2}$ & $\mathbf{1 1}$ & $\mathbf{9 0}$ \\
\hline
\end{tabular}

Results and Discussion:

Eighty percent (72/90) of the studied population belonged to the female gender. The distribution according to age groups is presented in table 1 , with $38 \%$ of persons (34/90) between 35-45 years. Almost one third were nurses (27/90), followed by physicians (26/90), medical auxiliars (21/90), laboratory technicians (8/90) and administrative assistants (8/90), as exposed in table 2 .

The table 2 also shows distribution characteristics by deparment. People from emergency room were the most participative with $34 \%(31 / 90)$.

Almost ninety-eight percent (88/90) of the population tested was negative for $\mathrm{IgG}$ pertussis toxin. The two equivocal results in a nurse (35-44 years, ER) and a physician ( $>45$ years, neonatology department) were probably due to cross-reactions.

\section{Results (cont.):}

Near 17\% (15/90) were not vaccinated against pertussis ( $>50$ years old) or, at least, did not have it registered on vaccination records. Four people loss their records. All the remaining (70/90) had the last booster 20 years ago or more, except one physician (25-34 years, ER) that received a booster of acellular vaccine in 2006 but he was also negative for IgG.

We found no relation between gender, age, professional category, years of vaccination and seroprevalence.

\section{Conclusions:}

This population is all susceptible to pertussis infection and a major reservoir for disease and for its transmission. A booster immunization should be recommended to these individuals. 